\section{The Difficult Asthma Patient}

\section{S1 BIOMARKERS IN ADULT ASTHMA: A SYSTEMATIC REVIEW OF 8-ISOPROSTANE IN EXHALED BREATH CONDENSATE}

${ }^{1} \mathrm{AM}$ Peel, ${ }^{1} \mathrm{CJ}$ Crossman-Barnes, ${ }^{1} \mathrm{~J}$ Tang, ${ }^{2} \mathrm{SJ}$ Fowler, ${ }^{3} \mathrm{GA}$ Davies, ${ }^{1} \mathrm{AM}$ Wilson, ${ }^{1}$ YK Loke. ${ }^{1}$ University of East Anglia, Norwich, UK; ${ }^{2}$ University Hospital South Manchester, Manchester, UK; ${ }^{3}$ Swansea University, Swansea, UK

\subsection{6/thoraxinl-2016-209333.7}

Introduction The potential of exhaled breath condensate (EBC) as a non-invasive indicator of airways disease has been studied for three decades or more. 8-isoprostane is a product of lipid peroxidation which can be detected within EBC. Studies have reported this as a potential objective indicator of oxidative stress in asthma. We therefore aimed to assess the evidence for the use of 8 -isoprostane in exhaled breath condensate (EBC) as a biomarker in adult asthma.

Design A systematic review and meta-analysis of EBC 8-isoprostane in asthma.

Methods We searched a number of online databases (including PubMed, Embase and Scopus) in January 2016. We included studies of adult non-smokers with EBC collection and asthma diagnosis conducted according to recognised guidelines. We aimed to pool data using random effects meta-analysis and assess heterogeneity using I2. Study quality and risk of bias was assessed using QUADAS-2 and GRADE.

Results We included twenty studies, the findings from which were inconsistent. Seven studies $(\mathrm{n}=329)$ reported 8-isoprostane concentrations in asthma to be significantly higher than that of control groups, whilst six studies $(n=403)$ did not. Only four studies had results appropriate for inclusion in a random effects meta-analysis of mean difference between asthma and controls (see Figure 1). This found a statistically significant betweengroups difference of $+22 \mathrm{pg} / \mathrm{ml}$ in asthma.

Confidence in the result is limited by the small number of studies; by substantial methodological and statistical heterogeneity $\left(\mathrm{I}^{2}=94\right)$; and by an inability to assess the risk of bias in key domains of the quality assessment tool.

Conclusion The clinical value of EBC 8-isoprostane as a quantitative assessment of oxidative stress in asthma remains unclear due to variability in results and methodological heterogeneity. It will be essential to develop accurate, reliable and standardised methods of both EBC collection and 8-isoprostane analysis if its use as a biomarker in asthma is to be evaluated.
S2 FRACTIONAL EXHALED NITRIC OXIDE (FENO) SUPPRESSION TO IDENTIFY NON-ADHERENCE IN DIFFICULT ASTHMA

${ }^{1} \mathrm{KJ}$ Hetherington, ${ }^{2} \mathrm{RW}$ Costello, ${ }^{1} \mathrm{LG}$ Heaney. 'Queens University Belfast, Belfast, UK; ${ }^{2}$ Royal College of Surgeons Ireland, Dublin, Ireland

\subsection{6/thoraxjnl-2016-209333.8}

Introduction Non-adherence, both intentional and non-intentional, is prevalent in 'difficult to manage' asthma and identification of patients who should respond to inhaled corticosteroids (ICS) is essential to reduce exacerbation risk and improve asthma control, but also to prevent inappropriate escalation of treatment (oral corticosteroids or biological therapies). Composite biomarker assessments (FeNO and blood eosinophils) have been shown to predict exacerbation risk in patients with asthma and these biomarkers also predict response to steroid treatment. It has previously been demonstrated that suppression of FeNO with directly observed ICS over 7 days can identify non-adherence to ICS treatment. The aim of this work was to further validate this test across UK severe asthma centres in the RASP-UK Consortium and to examine if the test can identify patients who should achieve good asthma control with better adherence to ICS treatment.

Methods Using remote monitoring technology (Vitalograph INCA $^{\mathrm{TM}}$ device and Aerocrine NIOX Vero), we developed a webbased interface to deliver FeNO suppression testing in RASP-UK Severe Asthma Centres. We examined the utility of FeNO suppression testing to predict inhaled steroid responsiveness and composite biomarker profile after 30 days of monitored optimised treatment on standard high dose ICS/LABA.

Results Forty of seventy patients had a positive suppression test. Using information from the Vitalograph INCA $^{\text {TM }}$ server to highlight technique and timing errors, these issues were addressed and patients proceeded to 30-day monitoring of high dose ICS/ LABA. A positive FeNO suppression test (Figure A) identified a biomarker-low population when adherent with high dose ICS ( $\geq 80 \%$ of inhaled steroid) whereas a negative test (Figure B) identified a biomarker-high population when adherent with high dose ICS.

Conclusion FeNO suppression testing is an effective means of identifying non-adherence to inhaled steroids in patients with difficult to manage asthma. This test can identify patients who should be optimised on inhaled steroids prior to consideration of treatment escalation.

Collaborating Centres Queen's University Belfast, University of Leicester, University of Glasgow, University of Oxford, The

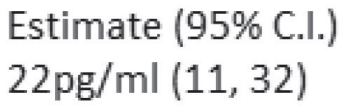

$1^{2}=94$

$P<0.001$

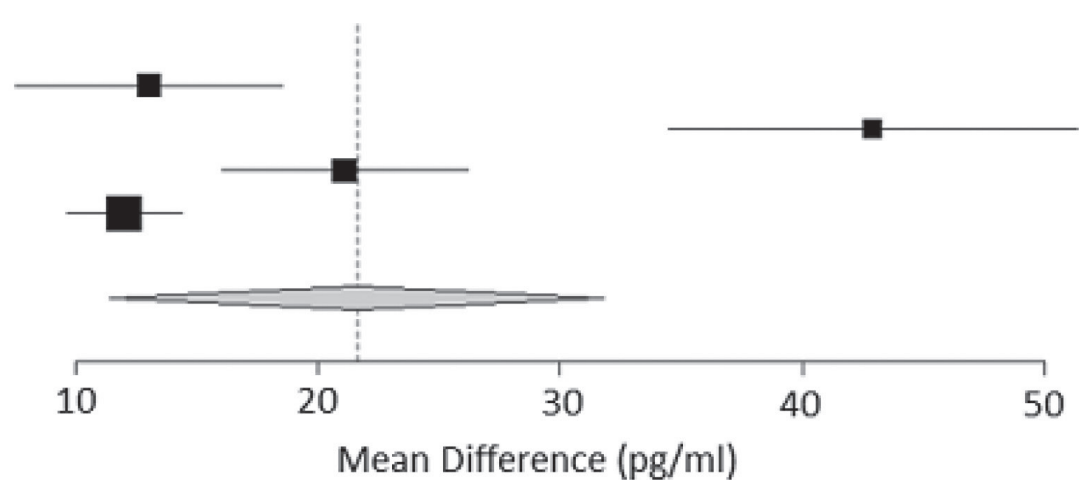

Mean Difference $(\mathrm{pg} / \mathrm{ml})$

Abstract S1 Figure 1 Random effects meta-analysis of mean between-group difference (asthma vs controls) 
Figure A

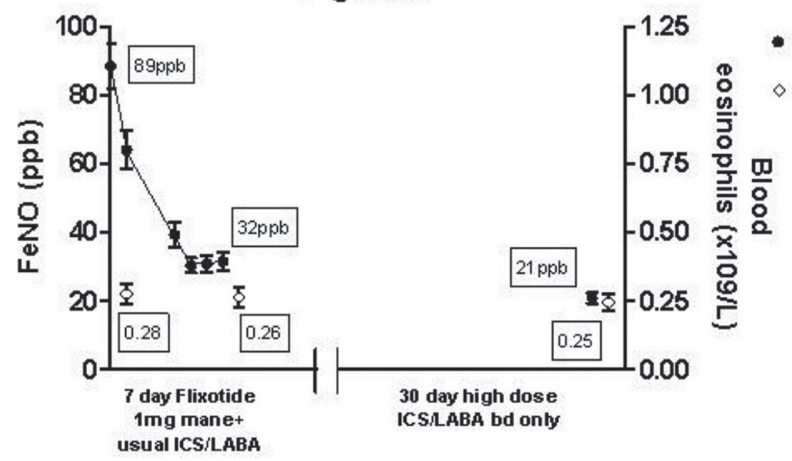

Figure B
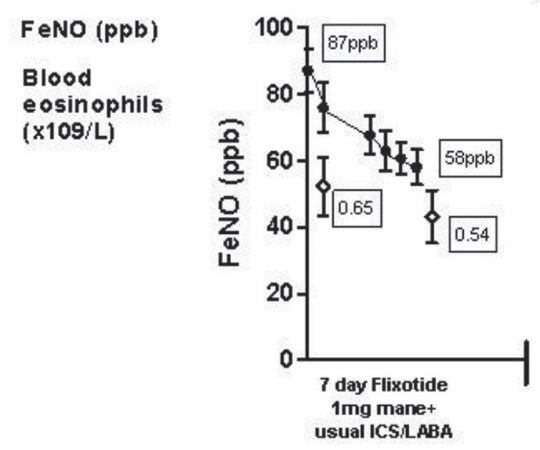

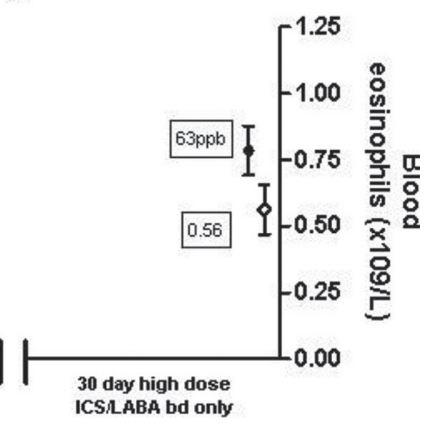

\section{Abstract S2 Figure 1}

University of Manchester, University of Birmingham, University College London.

\section{S3 THE UK'S LARGEST SEVERE ASTHMA MULTIDISCIPLINARY TEAM MEETING; EXPERIENCE FROM THE FIRST 18 MONTHS}

\begin{abstract}
${ }^{1} \mathrm{D}$ Ryan, ${ }^{1} \mathrm{R}$ Niven, ${ }^{2} \mathrm{H}$ Burhan, ${ }^{3} \mathrm{~J}$ Corless, ${ }^{1} S$ Diver, ${ }^{1} S$ Fowler, ${ }^{4} \mathrm{D}$ Menzies, ${ }^{5} \mathrm{R}$ O'Driscoll, ${ }^{6} \mathrm{~S}$ Scott, ${ }^{7} \mathrm{~N}$ Sehgal, ${ }^{8} \mathrm{~A}$ Vyas, ${ }^{7} \mathrm{D}$ Allen, ${ }^{9} \mathrm{~J}$ Blakey, ${ }^{1} \mathrm{~B}$ Kane. ${ }^{1}$ University Hospital of South Manchester NHS Foundation Trust, Manchester, UK; ${ }^{2}$ Royal Liverpool and Broadgreen University Hospitals NHS Trust, Liverpool, UK; ${ }^{3}$ Wirral University Teaching Hospital NHS Foundation Trust, Wirral, UK; ${ }^{4}$ Glan Clwyd Hospital, Rhyl, UK; ${ }^{5}$ Salford Royal NHS Foundation Trust, Salford, UK; ${ }^{6}$ Countess of Chester NHS Foundation Trust, Chester, UK; ${ }^{7}$ Pennine Acute Hospitals NHS Trust, Manchester, UK; ${ }^{8}$ Lancashire Teaching Hospitals NHS Foundation Trust, Preston, UK; ${ }^{9}$ Aintree University Hospital NHS Foundation Trust, Liverpool, UK
\end{abstract}

\subsection{6/thoraxjnl-2016-209333.9}

Background Severe asthma comprises 5\% of all asthma, but over $50 \%$ of the asthma healthcare burden. With multi-disciplinary team (MDT) working there is potential to improve patient outcomes and reduce healthcare costs. In 2013 NHS England produced service specifications for severe asthma aiming to develop a limited number of high volume specialist centres. In the North West we have developed a networked approach to specialised severe asthma services; the first Operation Delivery Network for a chronic disease. Representatives from 11 NHS Trusts and a central hub undertake a monthly virtual MDT meeting, with physicians, nurses, physiotherapists, clinical psychologists, speech and language therapists, allergists, pathologists and radiologists represented. All patients being considered for specialised treatments undergo MDT discussion for consensus approval of treatment.

Aim To summarise the experience and case-mix encountered during the first 18 months of operation of our regional virtual severe asthma MDT

Methods We reviewed all cases discussed at the MDT between January 2015 and June 2016. Cases were submitted online via nhs.net accounts, and data entered into a central database managed by two MDT coordinators for MDT discussion.

Results During this period 17 meetings were held, with 208 casesubmissions representing 185 patients, mean (SD) 12 (7) discussions per meeting. Indications for case submission included proposals for use of omalizumab, bronchial thermoplasty (BT), and steroid-sparing therapies, and for the discussion of patients with complex clinical issues, often managed across multiple sites. Omalizumab was approved in $81 \%$ of cases submitted, and BT in
$39 \%$, with more of the latter requiring multiple discussions $(30 \%$ versus 2\%) The most common reasons for non-approval of omalizumab were insufficient steroid requirement, poor adherence, and lack of allergy to a perennial allergen. Thermoplasty was not approved or listed for re-discussion for a variety of reasons, including 10 (43\%) that required further investigation.

Conclusion We describe our early experience of a multi-site virtual severe asthma MDT meeting facilitating expert care across a wide geographical area. This ensures governance in the use of novel and expensive severe asthma therapies, strengthens regional collaborations and ultimately aims to provide better patient care.

\section{S4 IMPLICATIONS OF GUIDANCE IN SCOTLAND ON ELIGIBILITY FOR TREATMENT WITH MEPOLIZUMAB AND OMALIZUMAB - AN IDEAL STUDY ANALYSIS}

CEA Hartmann, C Gait, NB Gunsoy, RA Mehta, FC Albers. GlaxoSmithKline, Research Triangle Park, USA

\subsection{6/thoraxjnl-2016-209333.10}

Rationale Severe asthma is a heterogeneous disease in which patients have diverse clinical characteristics and biomarkers, like eosinophils and IgE. It is important to understand their relationship in a severe asthma population. The IDEAL (Identification and Description of Severe Asthma Patients in a Cross-Sectional Study) study aimed to identify the proportion of patients with severe asthma who could be eligible for an anti IL-5 (mepolizu$\mathrm{mab}$ ) or anti-IgE (omalizumab) directed treatment, and those who may be eligible for either therapy.

Methods IDEAL, an observational study included subjects aged $\geq 12$ years with severe asthma defined according to ATS/ ERS guidelines by treatment with high-dose ICS plus additional controller(s) for $\geq 12$ months. Assessments included spirometry, a blood sample, and symptom/burden of illness questionnaires. Eligibility to mepolizumab and omalizumab were defined according to SMC advice (2016) and NICE MTA guidance (2013), which has been adopted in Scotland, respectively. Mepolizumab eligibility is defined as per SMC advice: patients who have eosinophils of at least 150 cells per microlitre $(0.15 \times 109 / \mathrm{L})$ at initiation of treatment and have had at least four asthma exacerbations in the preceding year or are receiving maintenance treatment with oral corticosteroids. Omalizumab eligibility (NICE MTA guidance) is defined as evidence of severe persistent allergic asthma and need for continuous or frequent treatment with oral corticosteroids 